\title{
Existence of Traveling Waves for a Delayed SIRS Epidemic Diffusion Model with Saturation Incidence Rate
}

\author{
Kai Zhou ${ }^{1}$ and Qi-Ru Wang ${ }^{2}$ \\ ${ }^{1}$ Department of Mathematics, Chizhou University, Chizhou, Anhui 247000, China \\ ${ }^{2}$ School of Mathematics and Computational Science, Sun Yat-sen University, Guangzhou 510275, China
}

Correspondence should be addressed to Qi-Ru Wang; mcswqr@mail.sysu.edu.cn

Received 22 January 2014; Accepted 11 April 2014; Published 30 April 2014

Academic Editor: Youyu Wang

Copyright ( 92014 K. Zhou and Q.-R. Wang. This is an open access article distributed under the Creative Commons Attribution License, which permits unrestricted use, distribution, and reproduction in any medium, provided the original work is properly cited.

This paper is concerned with the existence of traveling waves for a delayed SIRS epidemic diffusion model with saturation incidence rate. By using the cross-iteration method and Schauder's fixed point theorem, we reduce the existence of traveling waves to the existence of a pair of upper-lower solutions. By careful analyzsis, we derive the existence of traveling waves connecting the diseasefree steady state and the endemic steady state through the establishment of the suitable upper-lower solutions.

\section{Introduction}

Since Kermack and Mckendrick [1] proposed an ordinary differential system to study epidemiology in 1927, various models have been used to describe various kinds of epidemics, and the dynamics of these systems have been investigated. Let $S(t)$ represent the number of individuals who are susceptible to the disease, let $I(t)$ represent the number of infected individuals who are infectious and are able to spread the disease by contact with susceptible individuals, and let $R(t)$ represent the number of individuals who have been infected and then removed from the possibility of being infected again. Mena-Lorca and Hethcote [2] considered the following SIRS epidemic model:

$$
\begin{gathered}
\dot{S}(t)=A-d S(t)-\beta S(t) I(t)+\delta R(t), \\
\dot{I}(t)=\beta S(t) I(t)-(\gamma+\mu+d) I(t), \\
\dot{R}(t)=\gamma I(t)-(\delta+d) R(t),
\end{gathered}
$$

where the parameters $A, d, \beta, \delta, \gamma, \mu$ are positive constants and $A$ is the recruitment rate of the population, $d$ is the natural death rate of the population, $\beta$ is the transmission rate, $\delta$ is the rate at which recovered individuals lose immunity and return to the susceptible class, $\gamma$ is the recovery rate of the infective individuals, and $\mu$ is the death rate of the infective individuals due to disease. The SIRS model assumes that the recovered individuals have only temporary immunity, which is reasonable in the study of some communicable diseases.

However, due to the diseases latency or immunity, the presence of time delays in such models makes them more realistic. On the other hand, the environment in which an individual lives is actually heterogeneous and the mobility of people within a country or even worldwide is large; introducing the spatial diffusion in these epidemic models is unavoidable. In recent years, the dynamics of the delayed epidemic diffusion model have been widely studied by many researchers (see, e.g., [3-6]), and these studies are mainly focused on the global attractivity, basic reproductive number, and especially the epidemic waves. For example, Gan et al. [7] considered the following delayed SIRS epidemic model with spatial diffusion:

$$
\begin{aligned}
\frac{\partial S}{\partial t}= & D_{S} \frac{\partial^{2} S}{\partial x^{2}}+A-d S(x, t) \\
& -\beta S(x, t) I(x, t-\tau)+\delta R(x, t),
\end{aligned}
$$




$$
\begin{aligned}
\frac{\partial I}{\partial t}= & D_{I} \frac{\partial^{2} I}{\partial x^{2}}+\beta S(x, t) I(x, t-\tau) \\
& -(\gamma+\alpha+d) I(x, t), \\
\frac{\partial R}{\partial t}= & D_{R} \frac{\partial^{2} R}{\partial x^{2}}+\gamma I(x, t)-(\delta+d) R(x, t),
\end{aligned}
$$

and obtained the existence of traveling wave solutions.

In systems (1) and (2), the terms $\beta S(t) I(t)$ and $\beta S(x, t) I(x, t-\tau)$ are called incidence rate and both of them are bilinear. However, as the number of susceptible individuals is large, it is reasonable to consider the saturation incidence rate (see [8]) instead of the bilinear incidence rate. Motivated by the works mentioned above, we will consider the following delayed SIRS epidemic diffusion model with nonlinear saturation rate

$$
\begin{aligned}
\frac{\partial S}{\partial t}= & D_{S} \frac{\partial^{2} S}{\partial x^{2}}+A-d S(x, t) \\
& -\frac{\beta S(x, t) I(x, t-\tau)}{1+\alpha I(x, t-\tau)}+\delta R(x, t), \\
\frac{\partial I}{\partial t}= & D_{I} \frac{\partial^{2} I}{\partial x^{2}}+\frac{\beta S(x, t) I(x, t-\tau)}{1+\alpha I(x, t-\tau)} \\
& -(\gamma+\mu+d) I(x, t), \\
\frac{\partial R}{\partial t}= & D_{R} \frac{\partial^{2} R}{\partial x^{2}}+\gamma I(x, t)-(\delta+d) R(x, t)
\end{aligned}
$$

and study its traveling wave solutions. The main tool is the upper-lower solutions coupled with cross-iteration method established by $\mathrm{Ma}$ [9]. We point out that the nonlinear terms in (3) do not satisfy the common various (exponential) monotonicity conditions such as in [10-12]; thus the main difficulty is the construction and verification of the upperlower solutions.

\section{Preliminaries and Lemmas}

Throughout this paper, we employ the usual notations for the standard ordering in $\mathbb{R}^{3}$. That is, for $u=\left(u_{1}, u_{2}, u_{3}\right)$ and $v=$ $\left(v_{1}, v_{2}, v_{3}\right)$, we denote $u \leq v$ if $u_{i} \leq v_{i}, i=1,2,3 ; u<v$ if $u \leq v$ but $u \neq v$; and $u \ll v$ if $u \leq v$ but $u_{i} \neq v_{i}, i=1,2$, 3. Let $\|\cdot\|$ denote the Euclidean norm in $\mathbb{R}^{3}$.

First, we assume that $D_{S}=D_{I}=D_{R}=D$ for (3). Denoting $N=S+I+R$, then (3) reduces to the following system:

$$
\begin{aligned}
\frac{\partial N}{\partial t}= & D \frac{\partial^{2} N}{\partial x^{2}}+A-d N(x, t)-\mu I(x, t), \\
\frac{\partial I}{\partial t}= & D \frac{\partial^{2} I}{\partial x^{2}}+\frac{\beta(N-I-R) I(x, t-\tau)}{1+\alpha I(x, t-\tau)} \\
& -(\gamma+\mu+d) I(x, t), \\
\frac{\partial R}{\partial t}= & D \frac{\partial^{2} R}{\partial x^{2}}+\gamma I(x, t)-(\delta+d) R(x, t) .
\end{aligned}
$$

By making changes of variables $\widetilde{N}=A / d-N, \widetilde{I}=I, \widetilde{R}=$ $R$ and dropping the tildes, (4) is converted to the following system:

$$
\begin{gathered}
\frac{\partial N}{\partial t}=D \frac{\partial^{2} N}{\partial x^{2}}-d N(x, t)+\mu I(x, t) \\
\frac{\partial I}{\partial t}=D \frac{\partial^{2} I}{\partial x^{2}}+\beta\left(\frac{A}{d}-N-I-R\right) \frac{I(x, t-\tau)}{1+\alpha I(x, t-\tau)} \\
-(\gamma+\mu+d) I(x, t) \\
\frac{\partial R}{\partial t}=D \frac{\partial^{2} R}{\partial x^{2}}+\gamma I(x, t)-(\delta+d) R(x, t)
\end{gathered}
$$

Consider the equilibrium equation of system (5):

$$
\begin{gathered}
\mu I-d N=0, \\
\beta\left(\frac{A}{d}-N-I-R\right) \frac{I}{1+\alpha I}-(\gamma+\mu+d) I=0, \\
\gamma I-(\delta+d) R=0 .
\end{gathered}
$$

Obviously, system (5) often has a trivial equilibrium $E^{0}(0,0,0)$. From the first and the third equation of (6), we know that $N=(\mu / d) I, R=(\gamma /(\delta+d)) I$. Substituting the expressions into the second equation of (6), if $\mathfrak{R}_{0}:=A \beta / d(\gamma+\mu+d)>1$, we get a positive equilibrium $E^{*}\left(k_{1}, k_{2}, k_{3}\right)$ of system (5), where

$$
\begin{gathered}
k_{1}=\mu(\delta+d)[A \beta-d(\gamma+\mu+d)] \\
\times(d \beta[\delta(\mu+d)+d(\gamma+\mu+d)] \\
\left.\quad+d^{2} \alpha(\gamma+\mu+d)(\delta+d)\right)^{-1}, \\
k_{2}=\frac{(\delta+d)[A \beta-d(\gamma+\mu+d)]}{\beta[\delta(\mu+d)+d(\gamma+\mu+d)]+d \alpha(\gamma+\mu+d)(\delta+d)}, \\
k_{3}=\frac{\gamma[A \beta-d(\gamma+\mu+d)]}{\beta[\delta(\mu+d)+d(\gamma+\mu+d)]+d \alpha(\gamma+\mu+d)(\delta+d)} .
\end{gathered}
$$

By calculating, we can obtain that $k_{1}+k_{2}+k_{3}<A / d$, which is important in the following text. In fact,

$$
\begin{aligned}
k_{1}+ & k_{2}+k_{3} \\
= & \frac{[A \beta-d(\gamma+\mu+d)]}{\beta[\delta(\mu+d)+d(\gamma+\mu+d)]+d \alpha(\gamma+\mu+d)(\delta+d)} \\
& \times\left[\frac{\mu}{d}(\delta+d)+(\delta+d)+\gamma\right] \\
< & \frac{[A \beta-d(\gamma+\mu+d)]}{\beta\left(\mu \delta+\mu d+\delta d+\gamma d+d^{2}\right)} \\
& \times \frac{1}{d}\left(\mu \delta+\mu d+\delta d+\gamma d+d^{2}\right) \\
= & \frac{[A \beta-d(\gamma+\mu+d)]}{\beta d}<\frac{A}{d} .
\end{aligned}
$$


Now, we study the existence of traveling wave solutions for system (5) connecting $E^{0}$ and $E^{*}$.

Substituting $N(x, t)=\phi(x+c t), I(x, t)=\varphi(x+c t)$, $R(x, t)=\psi(x+c t)$ into (5), and denoting $x+c t$ still by $t$, we derive the following wave profile system from (5):

$$
\begin{aligned}
& D \phi^{\prime \prime}(t)-c \phi^{\prime}(t)-d \phi(t)+\mu \varphi(t)=0, \\
& D \varphi^{\prime \prime}(t)-c \varphi^{\prime}(t) \\
& \quad+\beta\left(\frac{A}{d}-\phi(t)-\varphi(t)-\psi(t)\right) \frac{\varphi(t-c \tau)}{1+\alpha \varphi(t-c \tau)} \\
& \quad-(\gamma+\mu+d) \varphi(t)=0, \\
& D \psi^{\prime \prime}(t)-c \psi^{\prime}(t)+\gamma \varphi(t)-(\delta+d) \psi(t)=0 .
\end{aligned}
$$

Note that $\mathfrak{R}_{0}>1$ imply $A \beta / d-(\gamma+\mu)>d$. Moreover, we have

$$
\mu \frac{k_{2}}{k_{1}}=d, \quad \gamma \frac{k_{2}}{k_{3}}-\delta=d .
$$

We can select suitable $M_{1}, M_{2}, M_{3}$ such that $M_{i}>k_{i}, i=$ $1,2,3$, which satisfy

$$
\begin{aligned}
& \frac{A \beta}{d}-(\gamma+\mu)>\mu \frac{M_{2}}{M_{1}}>d, \\
& \frac{A \beta}{d}-(\gamma+\mu)>\gamma \frac{M_{2}}{M_{3}}-\delta>d, \\
& \frac{A}{d}>M_{1}+M_{2}+M_{3} .
\end{aligned}
$$

Denote $C_{[\mathbf{0 , M}]}\left(\mathbb{R}, \mathbb{R}^{3}\right)=\left\{(\phi, \varphi, \psi) \in C\left(\mathbb{R}, \mathbb{R}^{3}\right): \mathbf{0} \leq\right.$ $(\phi(s), \varphi(s), \psi(s)) \leq \mathbf{M}\}$, where $\mathbf{M}=\left(M_{1}, M_{2}, M_{3}\right)$.

Denote $f=\left(f_{1}, f_{2}, f_{3}\right): C_{[0, \mathbf{M}]}\left(\mathbb{R}, \mathbb{R}^{3}\right) \rightarrow C\left(\mathbb{R}, \mathbb{R}^{3}\right):$

$$
\begin{aligned}
f_{1}(\phi, \varphi, \psi)(t)= & -d \phi(t)+\mu \varphi(t), \\
f_{2}(\phi, \varphi, \psi)(t)= & \beta\left(\frac{A}{d}-\phi(t)-\varphi(t)-\psi(t)\right) \\
& \times \frac{\varphi(t-c \tau)}{1+\alpha \varphi(t-c \tau)}-(\gamma+\mu+d) \varphi(t),
\end{aligned}
$$

$f_{3}(\phi, \varphi, \psi)(t)=\gamma \varphi(t)-(\delta+d) \psi(t)$. have

For $(\phi, \varphi, \psi) \in C_{[\mathbf{0}, \mathbf{M}]}\left(\mathbb{R}, \mathbb{R}^{3}\right)$, by a careful calculation, we

$$
\begin{aligned}
& \left|f_{1}\left(\phi_{1}, \varphi_{1}, \psi_{1}\right)(t)-f_{1}\left(\phi_{2}, \varphi_{2}, \psi_{2}\right)(t)\right| \\
& \quad \leq(d+\mu)|\Phi(t)-\Psi(t)|_{\mathbb{R}^{3}}, \\
& \left|f_{2}\left(\phi_{1}, \varphi_{1}, \psi_{1}\right)(t)-f_{2}\left(\phi_{2}, \varphi_{2}, \psi_{2}\right)(t)\right| \\
& \quad \leq L|\Phi(t)-\Psi(t)|_{\mathbb{R}^{3}}, \\
& \left|f_{3}\left(\phi_{1}, \varphi_{1}, \psi_{1}\right)(t)-f_{3}\left(\phi_{2}, \varphi_{2}, \psi_{2}\right)(t)\right| \\
& \quad \leq(\gamma+\delta+d)|\Phi(t)-\Psi(t)|_{\mathbb{R}^{3}},
\end{aligned}
$$

where $L:=A \beta / d+\beta\left(1+\alpha M_{2}^{2}+M_{1}+M_{3}+2 M_{2}+2 M_{2} /(1+\right.$ $\left.\left.\alpha M_{2}\right)\right)+(\gamma+\mu+d), \Phi=\left(\phi_{1}, \varphi_{1}, \psi_{1}\right), \Psi=\left(\phi_{2}, \varphi_{2}, \psi_{2}\right)$.

For the positive constants $\rho_{1}, \rho_{2}, \rho_{3}$, we define $H$ : $C_{[0, M]}\left(\mathbb{R}, \mathbb{R}^{3}\right) \rightarrow C\left(\mathbb{R}, \mathbb{R}^{3}\right)$ by

$$
\begin{aligned}
& H_{1}(\phi, \varphi, \psi)(t)=f_{1}(\phi, \varphi, \psi)(t)+\rho_{1} \phi(t), \\
& H_{2}(\phi, \varphi, \psi)(t)=f_{2}(\phi, \varphi, \psi)(t)+\rho_{2} \varphi(t), \\
& H_{3}(\phi, \varphi, \psi)(t)=f_{3}(\phi, \varphi, \psi)(t)+\rho_{3} \psi(t) .
\end{aligned}
$$

Then operators $H_{1}, H_{2}, H_{3}$ have the following properties.

Lemma 1. For $0 \leq \phi_{2}(t) \leq \phi_{1}(t) \leq M_{1}, 0 \leq \varphi_{2}(t) \leq \varphi_{1}(t) \leq$ $M_{2}, 0 \leq \psi_{2}(t) \leq \psi_{1}(t) \leq M_{3}$, one has

(i)

$$
\begin{aligned}
& H_{1}\left(\phi_{1}, \varphi_{1}, \psi_{1}\right)(t) \geq H_{1}\left(\phi_{2}, \varphi_{2}, \psi_{2}\right)(t), \\
& H_{3}\left(\phi_{1}, \varphi_{1}, \psi_{1}\right)(t) \geq H_{3}\left(\phi_{2}, \varphi_{2}, \psi_{2}\right)(t) ;
\end{aligned}
$$

(ii)

$$
\begin{aligned}
& H_{2}\left(\phi_{1}, \varphi_{1}, \psi_{1}\right)(t) \geq H_{2}\left(\phi_{1}, \varphi_{2}, \psi_{1}\right)(t), \\
& H_{2}\left(\phi_{2}, \varphi_{1}, \psi_{1}\right)(t) \geq H_{1}\left(\phi_{1}, \varphi_{1}, \psi_{1}\right)(t), \\
& H_{2}\left(\phi_{1}, \varphi_{1}, \psi_{2}\right)(t) \geq H_{2}\left(\phi_{1}, \varphi_{1}, \psi_{1}\right)(t) .
\end{aligned}
$$

Proof. According to the definitions of $f$ and $H$, we have

$$
\begin{aligned}
& H_{1}\left(\phi_{1}, \varphi_{1}, \psi_{1}\right)(t)-H_{1}\left(\phi_{2}, \varphi_{2}, \psi_{2}\right)(t) \\
& =\left(\rho_{1}-d\right)\left(\phi_{1}(t)-\phi_{2}(t)\right)+\mu\left(\varphi_{1}(t)-\varphi_{2}(t)\right), \\
& H_{3}\left(\phi_{1}, \varphi_{1}, \psi_{1}\right)(t)-H_{3}\left(\phi_{2}, \varphi_{2}, \psi_{2}\right)(t) \\
& =\left(\rho_{3}-d-\delta\right)\left(\psi_{1}(t)-\psi_{2}(t)\right) \\
& \quad+\gamma\left(\phi_{1}(t)-\phi_{2}(t)\right) .
\end{aligned}
$$

Let $\rho_{1}=d, \rho_{3}=d+\delta$; we obtain the properties for $H_{1}$ and $\mathrm{H}_{3}$.

For (ii), we have

$$
\begin{aligned}
H_{2}( & \left.\phi_{1}, \varphi_{1}, \psi_{1}\right)(t)-H_{2}\left(\phi_{1}, \varphi_{2}, \psi_{1}\right)(t) \\
= & \beta\left(\frac{A}{d}-\phi_{1}(t)-\psi_{1}(t)\right) \\
& \times\left(\frac{\varphi_{1}(t-c \tau)}{1+\alpha \varphi_{1}(t-c \tau)}-\frac{\varphi_{1}(t-c \tau)}{1+\alpha \varphi_{1}(t-c \tau)}\right) \\
& -\beta\left(\varphi_{1}(t) \frac{\varphi_{1}(t-c \tau)}{1+\alpha \varphi_{1}(t-c \tau)}-\varphi_{2}(t) \frac{\varphi_{2}(t-c \tau)}{1+\alpha \varphi_{2}(t-c \tau)}\right) \\
& +\left[\rho_{2}-(\gamma+\mu+d)\right]\left(\varphi_{1}(t)-\varphi_{2}(t)\right) .
\end{aligned}
$$

Note that $M_{1}+M_{3}<A / d$, and $x /(1+\alpha x)$ is nondecreasing; we have that the first term of the last formula is nonnegative, 
and the second term is bigger than $-\left(\beta M_{2} /\left(1+\alpha M_{2}\right)\right)\left(\varphi_{1}(t)-\right.$ $\left.\varphi_{2}(t)\right)$. Let $\rho_{2}=\beta M_{2} /\left(1+\alpha M_{2}\right)+\gamma+\mu+d$; we have $H_{2}\left(\phi_{1}, \varphi_{1}, \psi_{1}\right)(t) \geq H_{2}\left(\phi_{1}, \varphi_{2}, \psi_{1}\right)(t)$. Since

$$
\begin{aligned}
& H_{2}\left(\phi_{2}, \varphi_{1}, \psi_{1}\right)(t)-H_{2}\left(\phi_{1}, \varphi_{1}, \psi_{1}\right)(t) \\
& \quad=\left[\beta \frac{\varphi_{1}(t-c \tau)}{1+\alpha \varphi_{1}(t-c \tau)}+\rho_{2}\right]\left(\varphi_{1}(t)-\varphi_{2}(t)\right), \\
& H_{2}\left(\phi_{1}, \varphi_{1}, \psi_{2}\right)(t)-H_{2}\left(\phi_{1}, \varphi_{1}, \psi_{1}\right)(t) \\
& \quad=\left[\beta \frac{\varphi_{1}(t-c \tau)}{1+\alpha \varphi_{1}(t-c \tau)}+\rho_{2}\right]\left(\psi_{1}(t)-\psi_{2}(t)\right),
\end{aligned}
$$

then, for any positive constant $\rho_{2}$, we have $H_{2}\left(\phi_{2}, \varphi_{1}, \psi_{1}\right)(t) \geq$ $H_{1}\left(\phi_{1}, \varphi_{1}, \psi_{1}\right)(t), H_{2}\left(\phi_{1}, \varphi_{1}, \psi_{2}\right)(t) \geq H_{2}\left(\phi_{1}, \varphi_{1}, \psi_{1}\right)(t)$

Remark 2. For $\mathrm{H}_{2}$, we can further conclude that $H_{2}\left(\phi_{2}, \varphi_{1}, \psi_{2}\right)(t) \geq H_{2}\left(\phi_{1}, \varphi_{2}, \psi_{1}\right)(t)$ from Lemma 1(ii).

According to the definition of $H$, system (9) can be written as

$$
\begin{aligned}
& D \phi^{\prime \prime}(t)-c \phi^{\prime}(t)-\rho_{1} \phi(t)+H_{1}(\phi, \varphi, \psi)(t)=0, \\
& D \varphi^{\prime \prime}(t)-c \varphi^{\prime}(t)-\rho_{2} \varphi(t)+H_{2}(\phi, \varphi, \psi)(t)=0, \\
& D \psi^{\prime \prime}(t)-c \psi^{\prime}(t)-\rho_{3} \psi(t)+H_{3}(\phi, \varphi, \psi)(t)=0 .
\end{aligned}
$$

Define

$$
\begin{array}{ll}
\lambda_{1}=\frac{c-\sqrt{c^{2}+4 \rho_{1} D}}{2 D}, & \lambda_{2}=\frac{c+\sqrt{c^{2}+4 \rho_{1} D}}{2 D}, \\
\lambda_{3}=\frac{c-\sqrt{c^{2}+4 \rho_{2} D}}{2 D}, & \lambda_{4}=\frac{c+\sqrt{c^{2}+4 \rho_{2} D}}{2 D}, \\
\lambda_{5}=\frac{c-\sqrt{c^{2}+4 \rho_{3} D}}{2 D}, & \lambda_{6}=\frac{c+\sqrt{c^{2}+4 \rho_{3} D}}{2 D},
\end{array}
$$

and operator $F=\left(F_{1}, F_{2}, F_{3}\right): C_{[0, \mathbf{M}]}\left(\mathbb{R}, \mathbb{R}^{3}\right) \rightarrow C\left(\mathbb{R}, \mathbb{R}^{3}\right)$ by

$$
\begin{aligned}
& F_{1}(\phi, \varphi, \psi)(t) \\
& =\frac{1}{D\left(\lambda_{2}-\lambda_{1}\right)}\left[\int_{-\infty}^{t} e^{\lambda_{1}(t-s)} H_{1}(\phi, \varphi, \psi)(s) d s\right. \\
& \left.+\int_{t}^{+\infty} e^{\lambda_{2}(t-s)} H_{1}(\phi, \varphi, \psi)(s) d s\right], \\
& F_{2}(\phi, \varphi, \psi)(t) \\
& =\frac{1}{D\left(\lambda_{4}-\lambda_{3}\right)}\left[\int_{-\infty}^{t} e^{\lambda_{3}(t-s)} H_{2}(\phi, \varphi, \psi)(s) d s\right. \\
& \left.+\int_{t}^{+\infty} e^{\lambda_{4}(t-s)} H_{2}(\phi, \varphi, \psi)(s) d s\right],
\end{aligned}
$$

$$
\begin{aligned}
& F_{3}(\phi, \varphi, \psi)(t) \\
& =\frac{1}{D\left(\lambda_{6}-\lambda_{5}\right)}\left[\int_{-\infty}^{t} e^{\lambda_{5}(t-s)} H_{3}(\phi, \varphi, \psi)(s) d s\right. \\
& \left.+\int_{t}^{+\infty} e^{\lambda_{6}(t-s)} H_{3}(\phi, \varphi, \psi)(s) d s\right] .
\end{aligned}
$$

It is easy to see that $F_{i}(\phi, \varphi, \psi)(t)(i=1,2,3)$ satisfy system (20); thus the fixed point of operator $F$ satisfies (9), which is a traveling wave solution of system (5). Therefore, we will use Schauder's fixed point theorem to find the fixed point of $F$, where the continuity of $F$ is required. For this purpose, let $\nu>0$; we define a norm for $\Phi(t)=(\phi, \varphi, \psi)(t) \in C\left(\mathbb{R}, \mathbb{R}^{3}\right)$ by

$$
|\Phi|_{v}=\sup _{t \in \mathbb{R}} e^{-v|t|}\|\Phi(t)\|
$$

Define

$$
B_{\nu}\left(\mathbb{R}, \mathbb{R}^{3}\right)=\left\{\Phi \in C\left(\mathbb{R}, \mathbb{R}^{3}\right):|\Phi|_{\nu}<\infty\right\}
$$

Then it is obvious that $\left(B_{\nu}\left(\mathbb{R}, \mathbb{R}^{3}\right),|\cdot|_{\nu}\right)$ is a Banach space. We also need the following definition of upper and lower solutions for system (9).

Definition 3. A pair of continuous functions $\bar{\Phi}=(\bar{\phi}, \bar{\varphi}, \bar{\psi})$ and $\underline{\Phi}=(\phi, \varphi, \psi)$ are called an upper solution and a lower solution of $(\overline{9})$, respectively, if there exist finite points $T_{1}, T_{2}, \ldots, T_{m}$ such that $\bar{\Phi}, \underline{\Phi}$ are twice differentiable and bounded on $\mathbb{R} \backslash\left\{T_{i}\right\}, i=1,2, \ldots, m$, and satisfy

$$
\begin{aligned}
& D \bar{\phi}^{\prime \prime}(t)-c \bar{\phi}^{\prime}(t)+f_{1}(\bar{\phi}, \bar{\varphi}, \underline{\psi})(t) \leq 0, \\
& D \bar{\varphi}^{\prime \prime}(t)-c \bar{\varphi}^{\prime}(t)+f_{2}(\underline{\phi}, \bar{\varphi}, \underline{\psi})(t) \leq 0, \\
& D \bar{\psi}^{\prime \prime}(t)-c \bar{\psi}^{\prime}(t)+f_{3}(\bar{\phi}, \bar{\varphi}, \bar{\psi})(t) \leq 0, \\
& D \underline{\phi}^{\prime \prime}(t)-c \underline{\phi}^{\prime}(t)+f_{1}(\underline{\phi}, \underline{\varphi}, \underline{\psi})(t) \geq 0, \\
& D \underline{\varphi}^{\prime \prime}(t)-c \underline{\varphi}^{\prime}(t)+f_{2}(\bar{\phi}, \underline{\varphi}, \bar{\psi})(t) \geq 0, \\
& D \underline{\psi}^{\prime \prime}(t)-c \underline{\psi}^{\prime}(t)+f_{3}(\underline{\phi}, \underline{\varphi}, \underline{\psi})(t) \geq 0,
\end{aligned}
$$

for $t \in \mathbb{R} \backslash\left\{T_{i}\right\}$, respectively.

We assume that a pair of upper-lower solutions $\bar{\Phi}=$ $(\bar{\phi}, \bar{\varphi}, \bar{\psi})$ and $\underline{\Phi}=(\underline{\phi}, \underline{\varphi}, \underline{\psi})$ are given such that

(P1)

$$
\begin{aligned}
(0,0,0) & \leq(\underline{\phi}(t), \underline{\varphi}(t), \underline{\psi}(t)) \leq(\bar{\phi}(t), \bar{\varphi}(t), \bar{\psi}(t)) \\
& \leq\left(M_{1}, M_{2}, M_{3}\right) ;
\end{aligned}
$$


(P2)

$$
\begin{aligned}
& \lim _{t \rightarrow-\infty}(\underline{\phi}(t), \underline{\varphi}(t), \underline{\psi}(t)) \\
& =\lim _{t \rightarrow-\infty}(\bar{\phi}(t), \bar{\varphi}(t), \bar{\psi}(t))=(0,0,0), \\
& \lim _{t \rightarrow+\infty}(\underline{\phi}(t), \underline{\varphi}(t), \underline{\psi}(t)) \\
& \quad=\lim _{t \rightarrow+\infty}(\bar{\phi}(t), \bar{\varphi}(t), \bar{\psi}(t))=\left(k_{1}, k_{2}, k_{3}\right) ;
\end{aligned}
$$

(P3)

$$
\bar{\Phi}^{\prime}(t+) \leq \bar{\Phi}^{\prime}(t-), \quad \underline{\Phi}^{\prime}(t+) \geq \underline{\Phi}^{\prime}(t-) \quad \text { for } t \in \mathbb{R} .
$$

Define the set

$$
\begin{gathered}
\Gamma=\left\{(\phi, \varphi, \psi)(t) \in C_{[0, \mathbf{M}]}\left(\mathbb{R}, \mathbb{R}^{3}\right): \underline{\phi}(t) \leq \phi(t) \leq \bar{\phi}(t),\right. \\
\underline{\varphi}(t) \leq \varphi(t) \leq \bar{\varphi}(t), \underline{\psi}(t) \leq \psi(t) \leq \bar{\psi}(t)\} .
\end{gathered}
$$

Then by the property of $H$, we have the property of $F$.

Lemma 4. For $0 \leq \phi_{2}(t) \leq \phi_{1}(t) \leq M_{1}, 0 \leq \varphi_{2}(t) \leq \varphi_{1}(t) \leq$ $M_{2}, 0 \leq \psi_{2}(t) \leq \psi_{1}(t) \leq M_{3}$, one has

$$
\begin{aligned}
& F_{1}\left(\phi_{1}, \varphi_{1}, \psi_{1}\right)(t) \geq F_{1}\left(\phi_{2}, \varphi_{2}, \psi_{2}\right)(t), \\
& F_{2}\left(\phi_{2}, \varphi_{1}, \psi_{2}\right)(t) \geq F_{2}\left(\phi_{1}, \varphi_{2}, \psi_{1}\right)(t), \\
& F_{3}\left(\phi_{1}, \varphi_{1}, \psi_{1}\right)(t) \geq F_{3}\left(\phi_{2}, \varphi_{2}, \psi_{2}\right)(t) .
\end{aligned}
$$

Similar to Lemmas 4.4-4.6 in [7], we have the following lemmas and omit their proofs.

Lemma 5. $F=\left(F_{1}, F_{2}, F_{3}\right)$ is continuous with respect to the norm $|\cdot|_{\nu}$ in $\left(B_{\gamma}\left(\mathbb{R}, \mathbb{R}^{3}\right)\right)$.

Lemma 6. Consider $F: \Gamma \rightarrow \Gamma$.

Lemma 7. $F: \Gamma \rightarrow \Gamma$ is compact with respect to the norm $|\cdot|_{v}$.

Theorem 8. Assume that $A \beta / d(\gamma+\mu+d)>1$. If (9) has a pair of upper-lower solutions $\bar{\Phi}=(\bar{\phi}, \bar{\varphi}, \bar{\psi})$ and $\Phi=(\phi, \varphi, \psi)$ and satisfies (P1), (P2), and (P3), then system (5) has a traveling wave solution.

Proof. According to Lemmas 5-7 and applying Schauder's fixed point theorem, operator $F$ has a fixed point $\Phi^{*}=$ $\left(\phi^{*}, \varphi^{*}, \psi^{*}\right) \in \Gamma$, which is traveling wave for system (5). Furthermore, by (P2) we have

$$
\begin{aligned}
& \lim _{t \rightarrow-\infty}\left(\phi^{*}, \varphi^{*}, \psi^{*}\right)=(0,0,0), \\
& \lim _{t \rightarrow+\infty}\left(\phi^{*}, \varphi^{*}, \psi^{*}\right)=\left(k_{1}, k_{2}, k_{3}\right) .
\end{aligned}
$$

Therefore, the fixed point is a traveling wave solution for (5) connecting $E^{0}$ and $E^{*}$. The proof is complete.

\section{Existence of Traveling Waves}

To prove the existence of traveling wave solutions for (5), we only need to construct a pair of upper-lower solutions.

Consider the following functions:

$$
\begin{aligned}
& \Delta_{1}(\eta, c)=D \eta^{2}-c \eta-d+\mu \frac{M_{2}}{M_{1}}, \\
& \Delta_{2}(\eta, c)=D \eta^{2}-c \eta+\frac{A \beta}{d}-(\gamma+\mu+d), \\
& \Delta_{3}(\eta, c)=D \eta^{2}-c \eta-(\delta+d)+\gamma \frac{M_{2}}{M_{3}} .
\end{aligned}
$$

Note that (11) and $\mathfrak{R}_{0}>1$; we know that there exist positive numbers $c_{1}^{*}, c_{2}^{*}, c_{3}^{*}$ such that

$\Delta_{1}(\eta, c)=0$ has two zeros $0<\eta_{1}<\eta_{2}$, for $c>c_{1}^{*}$,

$\Delta_{2}(\eta, c)=0$ has two zeros $0<\eta_{3}<\eta_{4}$, for $c>c_{2}^{*}$,

$\Delta_{3}(\eta, c)=0$ has two zeros $0<\eta_{5}<\eta_{6}$, for $c>c_{3}^{*}$.

Denote $c^{*}=\max \left\{c_{1}^{*}, c_{2}^{*}, c_{3}^{*}\right\}$. According to [5, Lemma 3.8], we have $\eta_{1}<\eta_{3}$ and $\eta_{5}<\eta_{3}$.

Assume that $\mu / d+\gamma /(\delta+d)<1$; we can select $\varepsilon_{i}>0(i=$ $1,2, \ldots, 6), \varepsilon_{1}, \varepsilon_{2} \in\left(0, k_{1}\right), \varepsilon_{3}, \varepsilon_{4} \in\left(0, k_{2}\right), \varepsilon_{5}, \varepsilon_{6} \in\left(0, k_{3}\right)$ satisfying the following inequalities:

$$
\begin{gathered}
\mu\left(k_{2}+\varepsilon_{3}\right)-d\left(k_{1}+\varepsilon_{1}\right)<0, \\
\beta\left(\frac{A}{d}-k_{1}+\varepsilon_{2}-k_{2}-\varepsilon_{3}-k_{3}+\varepsilon_{6}\right) \\
\quad-(\gamma+\mu+d)<0, \\
\gamma\left(k_{2}+\varepsilon_{3}\right)-(\delta+d)\left(k_{3}+\varepsilon_{5}\right)<0, \\
d\left(k_{1}-\varepsilon_{2}\right)-\mu\left(k_{2}-\varepsilon_{4}\right)<0, \\
(\gamma+\mu+d)-\frac{\beta}{1+\alpha\left(k_{2}-\varepsilon_{4}\right)} \\
\quad \times\left(\frac{A}{d}-k_{1}-\varepsilon_{1}-k_{2}+\varepsilon_{4}-k_{3}-\varepsilon_{5}\right)<0, \\
(\delta+d)\left(k_{3}-\varepsilon_{6}\right)-\gamma\left(k_{2}-\varepsilon_{4}\right)<0 .
\end{gathered}
$$

In fact, we first choose $\varepsilon_{3}, \varepsilon_{4} \in\left(0, k_{2}\right)$ such that

$$
\left(\frac{\mu}{d}+\frac{\gamma}{\delta+d}\right) \varepsilon_{3}<\varepsilon_{4}, \quad\left(\frac{\mu}{d}+\frac{\gamma}{\delta+d}\right) \varepsilon_{4}<\varepsilon_{3} .
$$


For $\varepsilon_{3} \in\left(0, k_{2}\right)$, noting that $k_{1}=(\mu / d) k_{2}$ and $k_{3}=(\gamma /(\delta+$ d)) $k_{2}$, we can find $\varepsilon_{1} \in\left(0, k_{1}\right), \varepsilon_{5} \in\left(0, k_{3}\right)$ such that

$$
\begin{aligned}
k_{1}>\varepsilon_{1}>\frac{\mu}{d} \varepsilon_{3} & =\frac{\mu}{d}\left(k_{2}+\varepsilon_{3}\right)-k_{1} \\
& \Longrightarrow \mu\left(k_{2}+\varepsilon_{3}\right)-d\left(k_{1}+\varepsilon_{1}\right)<0, \\
k_{3}>\varepsilon_{5}>\frac{\gamma}{\delta+d} \varepsilon_{3} & =\frac{\gamma}{\delta+d}\left(k_{2}+\varepsilon_{3}\right)-k_{3} \\
& \Longrightarrow \gamma\left(k_{2}+\varepsilon_{3}\right)-(\delta+d)\left(k_{3}+\varepsilon_{5}\right)<0 .
\end{aligned}
$$
that

For $\varepsilon_{4} \in\left(0, k_{2}\right)$, we can find $\varepsilon_{2} \in\left(0, k_{1}\right), \varepsilon_{6} \in\left(0, k_{3}\right)$ such

$$
\begin{aligned}
k_{1}>\varepsilon_{2}>\frac{\mu}{d} \varepsilon_{4}= & k_{1}-\frac{\mu}{d}\left(k_{2}-\varepsilon_{4}\right) \\
\Longrightarrow & d\left(k_{1}-\varepsilon_{2}\right)-\mu\left(k_{2}-\varepsilon_{4}\right)<0, \\
k_{3}>\varepsilon_{6}>\frac{\gamma}{\delta+d} \varepsilon_{4} & =k_{3}-\frac{\gamma}{\delta+d}\left(k_{2}-\varepsilon_{4}\right) \\
& \Longrightarrow(\delta+d)\left(k_{3}-\varepsilon_{6}\right)-\gamma\left(k_{2}-\varepsilon_{4}\right)<0 .
\end{aligned}
$$

Furthermore, for $\varepsilon_{1}>(\mu / d) \varepsilon_{3}, \varepsilon_{5}>(\gamma /(\delta+d)) \varepsilon_{3}$ and (35), we can find suitable $\varepsilon_{1}, \varepsilon_{5}$ satisfying $\varepsilon_{1}+\varepsilon_{5}<\varepsilon_{4}$. Similarly, we can find suitable $\varepsilon_{2}, \varepsilon_{6}$ satisfying $\varepsilon_{2}+\varepsilon_{6}<\varepsilon_{3}$. Thus we have

$$
\begin{aligned}
& \beta\left(\frac{A}{d}-k_{1}+\varepsilon_{2}-k_{2}-\varepsilon_{3}-k_{3}+\varepsilon_{6}\right)-(\gamma+\mu+d)<0 \\
& (\gamma+\mu+d)-\frac{\beta}{1+\alpha\left(k_{2}-\varepsilon_{4}\right)} \\
& \quad \times\left(\frac{A}{d}-k_{1}-\varepsilon_{1}-k_{2}+\varepsilon_{4}-k_{3}-\varepsilon_{5}\right)<0 .
\end{aligned}
$$

We define continuous functions $\Phi(t)$ = $\left(\phi_{1}(t), \varphi_{1}(t), \psi_{1}(t)\right)$ and $\Psi(t)=\left(\phi_{2}(t), \varphi_{2}(t), \psi_{2}(t)\right)$ as follows:

$$
\begin{aligned}
& \phi_{1}(t)= \begin{cases}k_{1} e^{\eta_{1} t}, & t \leq t_{1}, \\
k_{1}+\varepsilon_{1} e^{-\eta t}, & t>t_{1},\end{cases} \\
& \phi_{2}(t)= \begin{cases}0, & t \leq t_{2}, \\
k_{1}-\varepsilon_{2} e^{-\eta t}, & t>t_{2},\end{cases} \\
& \varphi_{1}(t)= \begin{cases}k_{2} e^{\eta_{3} t}, \\
k_{2}+\varepsilon_{3} e^{-\eta t}, & t>t_{3},\end{cases} \\
& \varphi_{2}(t)= \begin{cases}0, & t \leq t_{4}, \\
k_{2}-\varepsilon_{4} e^{-\eta t}, & t>t_{4},\end{cases} \\
& \psi_{1}(t)= \begin{cases}k_{3} e^{\eta_{5} t}, & t \leq t_{5}, \\
k_{3}+\varepsilon_{5} e^{-\eta t}, & t>t_{5},\end{cases} \\
& \psi_{2}(t)= \begin{cases}0, & t \leq t_{6}, \\
k_{3}-\varepsilon_{6} e^{-\eta t}, & t>t_{6},\end{cases}
\end{aligned}
$$

where $t_{1}, t_{3}, t_{5}>0, t_{2}, t_{4}, t_{6}<0$, and $\eta>0$ is a proper constant to be chosen later.

Furthermore, we can conclude that $t_{3} \geq \max \left\{t_{1}, t_{5}\right\}$ and $t_{4} \leq \min \left\{t_{2}, t_{6}\right\}$, which can help us verify the upper-lower solution for system (9). We point out that $\Phi(t)$ and $\Psi(t)$ satisfy (P1), (P2), and (P3) for proper parameters.

Lemma 9. Suppose $\mu / d+\gamma /(\delta+d)<1$. Then the functions $\Phi(t)$ and $\Psi(t)$ defined above are upper and lower solutions of (9), respectively.

Proof. If $t \leq t_{1}, \phi_{1}(t)=k_{1} e^{\eta_{1} t}, \varphi_{1}(t)=k_{2} e^{\eta_{3} t}$, we have

$$
\begin{aligned}
p_{1}(t) & :=D \phi_{1}^{\prime \prime}(t)-c \phi_{1}^{\prime}(t)-d \phi_{1}(t)+\mu \varphi_{1}(t) \\
& \leq\left(D \eta_{1}^{2}-c \eta_{1}-d+\mu \frac{M_{2}}{M_{1}}\right) k_{1} e^{\eta_{1} t}=0 .
\end{aligned}
$$

If $t>t_{1}, \phi_{1}(t)=k_{1}+\varepsilon_{1} e^{-\eta t}, \varphi_{1}(t) \leq k_{2}+\varepsilon_{3} e^{-\eta t}$, we know

$$
p_{1}(t) \leq I_{1}(\eta)
$$

where $I_{1}(\eta)=\left(D \varepsilon_{1} \eta^{2}+c \varepsilon_{1} \eta\right) e^{-\eta t}-d\left(k_{1}+\varepsilon_{1} e^{-\eta t}\right)+\mu\left(k_{2}+\right.$ $\left.\varepsilon_{3} e^{-\eta t}\right)$. Then $I_{1}(0)=\mu\left(k_{2}+\varepsilon_{3}\right)-d\left(k_{1}+\varepsilon_{1}\right)$. It follows from (34) that $I_{1}(0)<0$ and there exists $\eta_{1}^{*}>0$ such that $p_{1}(t)<0$ for all $\eta \in\left(0, \eta_{1}^{*}\right)$.

$$
\text { If } t \leq t_{3}, \varphi_{1}(t)=k_{2} e^{\eta_{3} t} \text {, we obtain that }
$$

$$
\begin{aligned}
p_{2}(t):= & D \varphi_{1}^{\prime \prime}(t)-c \varphi_{1}^{\prime}(t) \\
& +\beta\left(\frac{A}{d}-\phi_{2}(t)-\varphi_{1}(t)-\psi_{2}(t)\right) \frac{\varphi_{1}(t-c \tau)}{1+\alpha \varphi_{1}(t-c \tau)} \\
& -(\gamma+\mu+d) \varphi_{1}(t) \\
\leq & \left(D \eta_{2}^{3}-c \eta_{3}-\gamma-\mu-d\right) k_{2} e^{\eta_{3} t}+\frac{A \beta}{d} k_{2} e^{\eta_{3}(t-c \tau)} \\
\leq & {\left[D \eta_{2}^{3}-c \eta_{3}+\frac{A \beta}{d}-\gamma-\mu-d\right] k_{2} e^{\eta_{3} t}=0 . }
\end{aligned}
$$

If $t>t_{3}, \varphi_{1}(t)=k_{2}+\varepsilon_{3} e^{-\eta t}, \phi_{2}(t)=k_{1}-\varepsilon_{2} e^{-\eta t}, \psi_{2}(t)=$ $k_{3}-\varepsilon_{6} e^{-\eta t}$, we have

$$
p_{2}(t) \leq I_{2}(\eta)
$$

where

$$
\begin{aligned}
I_{2}(\eta)= & \left(D \varepsilon_{3} \eta^{2}+c \varepsilon_{3} \eta\right) e^{-\eta t} \\
& -(\gamma+\mu+d)\left(k_{2}+\varepsilon_{3} e^{-\eta t}\right) \\
& +\beta\left[\frac{A}{d}-k_{1}+\varepsilon_{2} e^{-\eta t}-k_{2}-\varepsilon_{3} e^{-\eta t}-k_{3}+\varepsilon_{6} e^{-\eta t}\right] \\
& \times\left(k_{2}+\varepsilon_{3} e^{-\eta(t-c \tau)}\right) .
\end{aligned}
$$

It follows from (34) that $I_{2}(0)<0$ and there exists $\eta_{2}^{*}>0$ such that $p_{2}(t)<0$ for all $\eta \in\left(0, \eta_{2}^{*}\right)$. 


$$
\begin{aligned}
& \text { If } t \leq t_{5}, \psi_{1}(t)=k_{3} e^{\eta_{5} t}, \varphi_{1}(t)=k_{2} e^{\eta_{3} t}, \text { we have } \\
& \begin{aligned}
p_{3}(t) & :=D \psi_{1}^{\prime \prime}(t)-c \psi_{1}^{\prime}(t)+\gamma \varphi_{1}(t)-(\delta+d) \psi_{1}(t) \\
& \leq\left[D \eta_{5}^{2}-c \eta_{5}-(\delta+d)+\gamma \frac{M_{2}}{M_{3}}\right] k_{3} e^{\eta_{5} t}=0 .
\end{aligned}
\end{aligned}
$$

If $t>t_{5}, \psi_{1}(t)=k_{3}+\varepsilon_{5} e^{-\eta t}, \varphi_{1}(t) \leq k_{2}+\varepsilon_{3} e^{-\eta t}$,

$$
p_{3}(t) \leq I_{3}(\eta)
$$

where $I_{3}(\eta)=\left(D \varepsilon_{5} \eta^{2}+c \varepsilon_{5} \eta\right) e^{-\eta t}+\gamma\left(k_{2}+\varepsilon_{3} e^{-\eta t}\right)-(\delta+d)\left(k_{3}+\right.$ $\left.\varepsilon_{5} e^{-\eta t}\right)$. We can derive from (34) that there exists $\eta_{3}^{*}>0$ such that $p_{3}(t)<0$ for all $\eta \in\left(0, \eta_{3}^{*}\right)$.

If $t \leq t_{2}, \phi_{2}(t)=0$, we have

$$
\begin{aligned}
q_{1}(t):= & D \phi_{2}^{\prime \prime}(t)-c \phi_{2}^{\prime}(t)-d \phi_{2}(t) \\
& +\mu \varphi_{2}(t)=\mu \varphi_{2}(t) \geq 0 .
\end{aligned}
$$

If $t>t_{2}, \phi_{2}(t)=k_{1}-\varepsilon_{2} e^{-\eta t}, \varphi_{2}(t)=k_{2}-\varepsilon_{4} e^{-\eta t}$, we have

$$
q_{1}(t) \geq I_{4}(\eta)
$$

where $I_{4}(\eta)=-\left(D \varepsilon_{2} \eta^{2}+c \varepsilon_{2} \eta\right) e^{-\eta t}-d\left(k_{1}-\varepsilon_{2} e^{-\eta t}\right)+\mu\left(k_{2}-\right.$ $\left.\varepsilon_{4} e^{-\eta t}\right)$. It follows from (34) that $I_{4}(0)>0$ and there exists $\eta_{4}^{*}>0$ such that $q_{1}(t)>0$ for all $\eta \in\left(0, \eta_{4}^{*}\right)$.

If $t \leq t_{4}, \varphi_{2}(t)=0$, we obtain that

$$
\begin{aligned}
q_{2}(t):= & D \varphi_{2}^{\prime \prime}(t)-c \varphi_{2}^{\prime}(t) \\
& +\beta\left(\frac{A}{d}-\phi_{1}(t)-\varphi_{2}(t)-\psi_{1}(t)\right) \frac{\varphi_{2}(t-c \tau)}{1+\alpha \varphi_{2}(t-c \tau)} \\
& -(\gamma+\mu+d) \varphi_{2}(t)=0 .
\end{aligned}
$$

If $t>t_{4}, \varphi_{2}(t)=k_{2}-\varepsilon_{4} e^{-\eta t}, \varphi_{2}(t-c \tau) \geq k_{2}-\varepsilon_{4} e^{-\eta(t-c \tau)}$, $\phi_{1}(t) \leq k_{1}+\varepsilon_{1} e^{-\eta t}, \psi_{1}(t) \leq k_{3}+\varepsilon_{5} e^{-\eta t}$, we have

$$
q_{2}(t) \geq I_{5}(\eta)
$$

where

$$
\begin{aligned}
I_{5}(\eta)= & -\left(D \varepsilon_{4} \eta^{2}+c \varepsilon_{4} \eta\right) e^{-\eta t} \\
& -(\gamma+\mu+d)\left(k_{2}-\varepsilon_{4} e^{-\eta t}\right) \\
& +\beta\left(\frac{A}{d}-k_{1}-\varepsilon_{1} e^{-\eta t}-k_{2}+\varepsilon_{4} e^{-\eta t}-k_{3}\right. \\
& \left.-\varepsilon_{5} e^{-\eta t}\right) \frac{k_{2}-\varepsilon_{4} e^{-\eta(t-c \tau)}}{1+\alpha k_{2}-\alpha \varepsilon_{4} e^{-\eta(t-c \tau)}} .
\end{aligned}
$$

It follows from (34) that $I_{5}(0)>0$ and there exists $\eta_{5}^{*}>0$ such that $q_{2}(t)>0$ for all $\eta \in\left(0, \eta_{5}^{*}\right)$.

If $t \leq t_{6}, \psi_{2}(t)=0$, we have

$$
\begin{aligned}
q_{3}(t):= & D \psi_{2}^{\prime \prime}(t)-c \psi_{2}^{\prime}(t)+\gamma \varphi_{2}(t) \\
& -(\delta+d) \psi_{2}(t) \geq 0
\end{aligned}
$$

If $t>t_{6}, \psi_{2}(t)=k_{3}-\varepsilon_{6} e^{-\eta t}, \varphi_{2}(t)=k_{2}-\varepsilon_{4} e^{-\eta t}$, we have

$$
q_{3}(t) \geq I_{6}(\eta)
$$

where $I_{6}(\eta)=-\left(D \varepsilon_{6} \eta^{2}+c \varepsilon_{6} \eta\right) e^{-\eta t}+\gamma\left(k_{2}-\varepsilon_{4} e^{-\eta t}\right)-(\delta+$ d) $\left(k_{3}-\varepsilon_{6} e^{-\eta t}\right)$. It follows from (34) that $I_{6}(0)>0$ and there exists $\eta_{6}^{*}>0$ such that $q_{3}(t)>0$ for all $\eta \in\left(0, \eta_{6}^{*}\right)$.

Thus, taking $\eta \in\left(0, \min _{1 \leq i \leq 6}\left\{\eta_{i}^{*}\right\}\right)$, we prove that $\Phi(t)$ and $\Psi(t)$ are upper and lower solutions of (9).

Now we obtain and state the main result in this paper.

Theorem 10. Assume that $A \beta / d(\gamma+\mu+d)>1$ and $\mu / d+$ $\gamma /(\delta+d)<1$; then, for any $c>c^{*}$, (5) has a traveling wave solution connecting two equilibria $E^{0}$ and $E^{*}$. Furthermore, system (4) has a traveling wave solution with speed $c$, which connects two states $(A / d, 0,0)$ and $\left(A / d-k_{1}, k_{2}, k_{3}\right)$.

\section{Conflict of Interests}

The authors declare that there is no conflict of interests regarding the publication of this paper.

\section{Acknowledgments}

Kai Zhou is supported by Scientific Research Program of Anhui Provincial Education Department (no. KJ2013B173). Qi-Ru Wang is supported by the NNSF of China (no. 11271379).

\section{References}

[1] M. Kermack and A. Mckendrick, "Contributions to the mathematical theory of epidemics," Proceedings of the Royal Society of London A, vol. 115, no. 4, pp. 700-721, 1927.

[2] J. Mena-Lorca and H. W. Hethcote, "Dynamic models of infectious diseases as regulators of population sizes," Journal of Mathematical Biology, vol. 30, no. 7, pp. 693-716, 1992.

[3] X.-S. Wang, H. Wang, and J. Wu, "Traveling waves of diffusive predator-prey systems: disease outbreak propagation,” Discrete and Continuous Dynamical Systems A, vol. 32, no. 9, pp. 33033324, 2012.

[4] P. Weng and X.-Q. Zhao, "Spreading speed and traveling waves for a multi-type SIS epidemic model," Journal of Differential Equations, vol. 229, no. 1, pp. 270-296, 2006.

[5] X. Yu, C. Wu, and P. X. Weng, "Traveling waves for a SIRS model with nonlocal diffusion," International Journal of Biomathematics, vol. 5, no. 5, Article ID 1250036, 2012.

[6] S. Zhang and R. Xu, "Travelling waves and global attractivity of an SIRS disease model with spatial diffusion and temporary immunity," Applied Mathematics and Computation, vol. 224, no. 1, pp. 635-651, 2013.

[7] Q. T. Gan, R. Xu, and P. H. Yang, "Travelling waves of a delayed SIRS epidemic model with spatial diffusion," Nonlinear Analysis: Real World Applications, vol. 12, no. 1, pp. 52-68, 2011.

[8] J. Yang, S. Liang, and Y. Zhang, “Travelling waves of a delayed SIR epidemic model with nonlinear incidence rate and spatial diffusion," PLoS ONE, vol. 6, no. 6, Article ID e21128, 2011.

[9] S. Ma, "Traveling wavefronts for delayed reaction-diffusion systems via a fixed point theorem," Journal of Differential Equations, vol. 171, no. 2, pp. 294-314, 2001. 
[10] J.-H. Huang and X.-F. Zou, "Travelling wave solutions in delayed reaction diffusion systems with partial monotonicity," Acta Mathematicae Applicatae Sinica (English Series), vol. 22, no. 2, pp. 243-256, 2006.

[11] W.-T. Li, G. Lin, and S. Ruan, "Existence of travelling wave solutions in delayed reaction-diffusion systems with applications to diffusion-competition systems," Nonlinearity, vol. 19, no. 6, pp. 1253-1273, 2006.

[12] Q.-R. Wang and K. Zhou, "Traveling wave solutions in delayed reaction-diffusion systems with mixed monotonicity", Journal of Computational and Applied Mathematics, vol. 233, no. 10, pp. 2549-2562, 2010. 


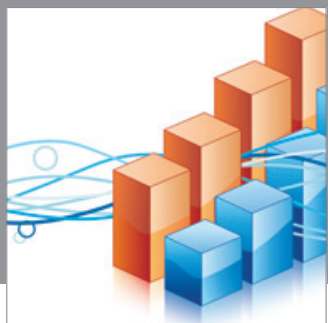

Advances in

Operations Research

mansans

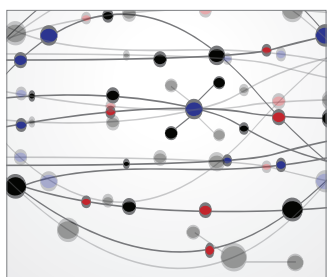

The Scientific World Journal
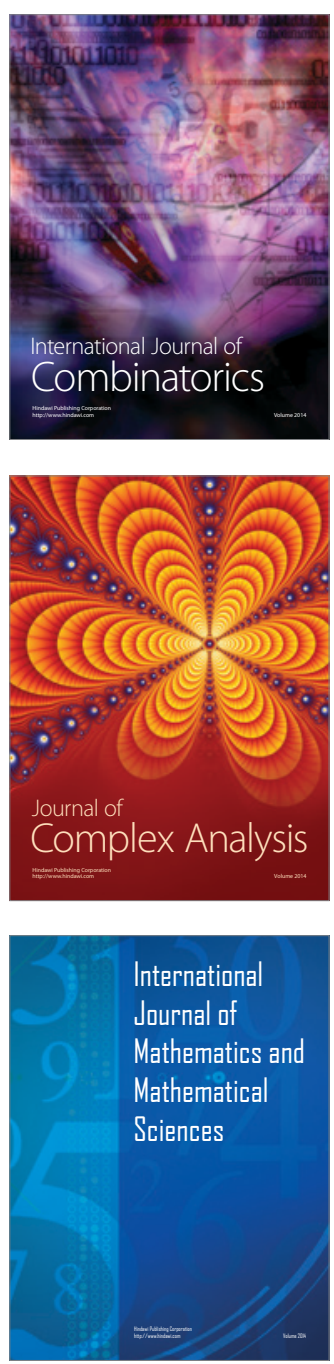
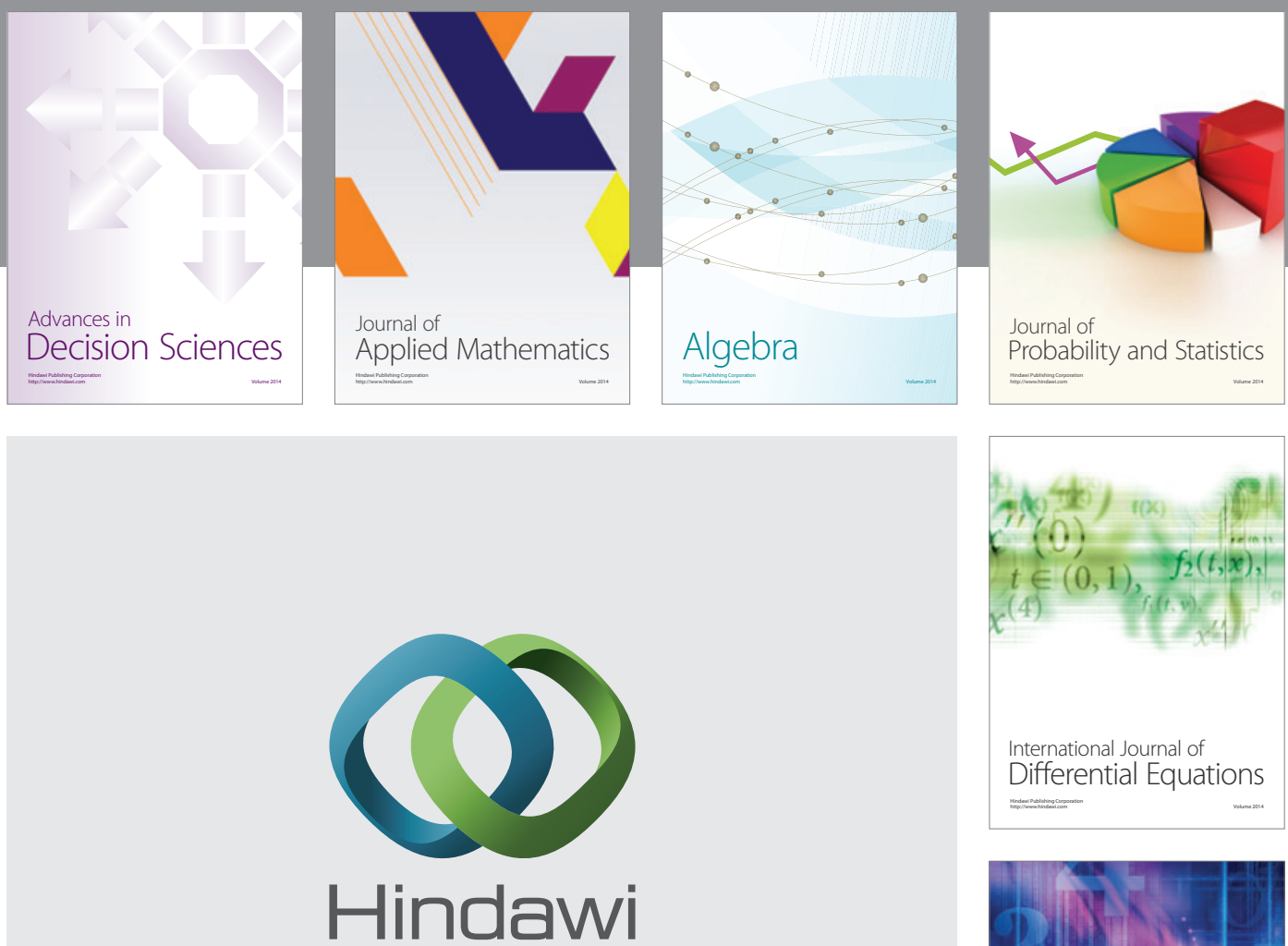

Submit your manuscripts at http://www.hindawi.com
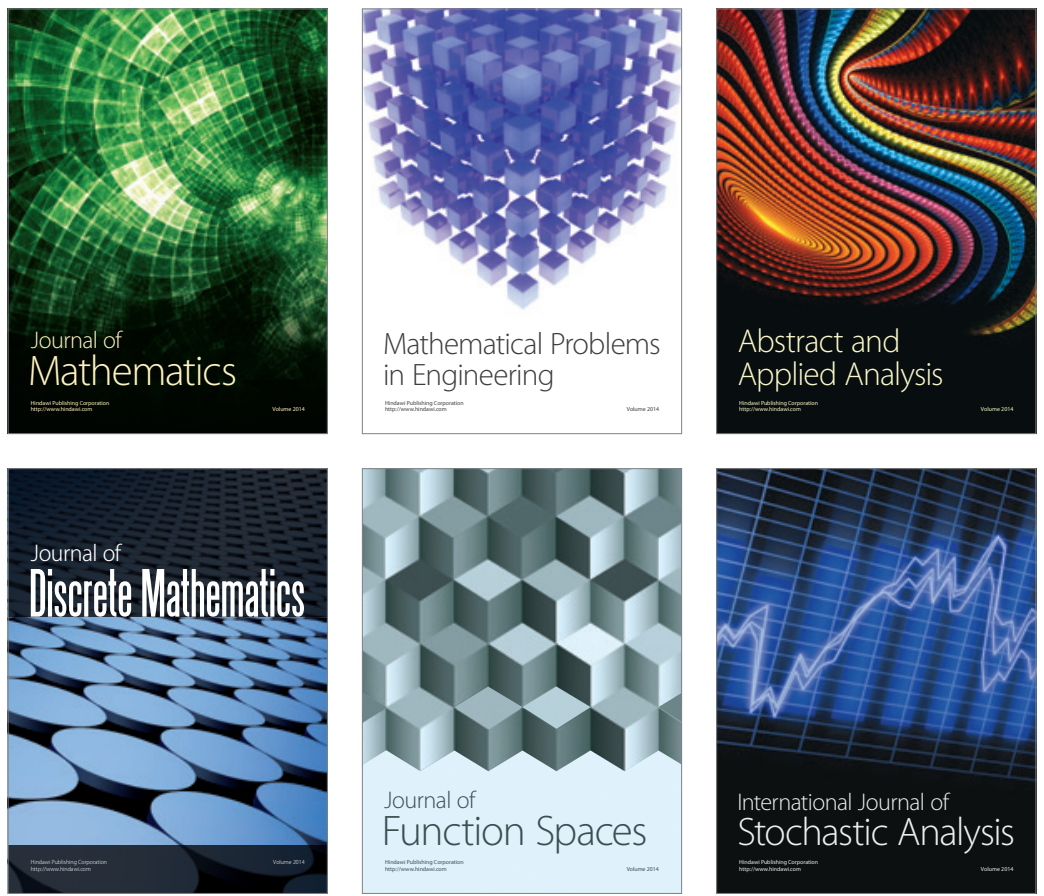

Journal of

Function Spaces

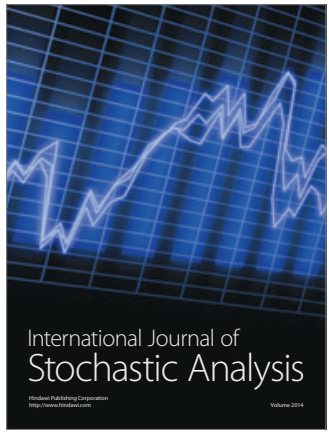

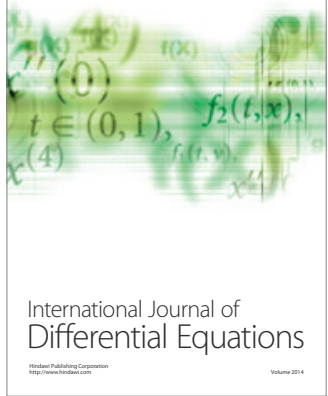
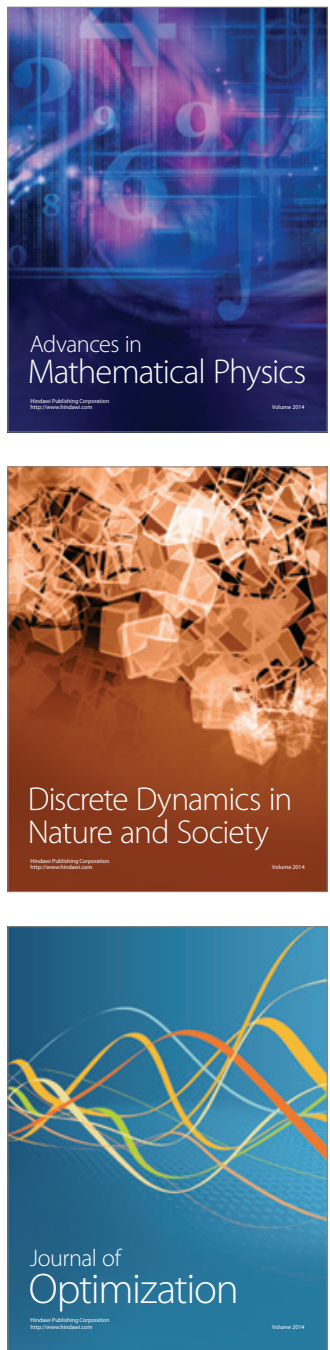